\title{
A DFT Study of Heteroatom Doped-Pyrazine as an Anode in Sodium ion Batteries
}

\author{
S.Z.J. Zaidi ${ }^{*}$, M. Raza ${ }^{1}$, S. Hassan ${ }^{2}$, C. Harito ${ }^{3}$ and F.C. Walsh ${ }^{4}$ \\ ${ }^{1}$ Institute of Chemical Engineering and Technology, University of the Punjab, 54000, Lahore, Punjab, Pakistan \\ ${ }^{2}$ School of Mechanical Engineering, Faculty of Engineering and Physical Sciences, University of Southampton, United \\ Kingdom \\ ${ }^{3}$ Industrial Engineering Department, Faculty of Engineering, Bina Nusantara University, Jakarta, Indonesia 11480 \\ ${ }^{4}$ Electrochemical Engineering Laboratory, Faculty of Engineering and Physical Sciences, University of Southampton, \\ Southampton, UK.
}

Corresponding Author Email: zohaib.icet@ pu.edu.pk

\begin{abstract}
Lithium ion batteries cannot satisfy increasing demand for energy storage. A range of complementary batteries are needed which are environmentally acceptable, of moderate cost and easy to manufacture/recycle. In this case, we have chosen pyrazine to be used in the sodium ion batteries to meet the energy storage requirements of tomorrow. Pyrazine is studied as a possible anode material for bio-batteries, lithiumion, and sodium ion batteries due to its broad set of useful properties such as ease of synthesis, low cost, ability to be charge-discharge cycled, and stability in the electrolyte. The heteroatom doped-pyrazine with atoms of boron, fluorine, phosphorous, and sulphur as an anode in sodium ion batteries has improved the stability and intercalation of sodium ions at the anode. The longest bond observed between sodium ion and sulphur-doped pyrazine at $2.034 \AA$ A. The electronic charge is improved and further enhanced by the presence of highly electronegative atoms such as fluorine and bromine in an already electron-attracting pyrazine compound. The highest adsorption energy is observed for the borondoped pyrazine at $-2.735 \mathrm{eV}$. The electron-deficient sites present in fluorine and bromine help in improving the electronic storage of the sodium ion batteries. A mismatch is observed between the adsorption energy and bond length in pyrazine doped with fluorine and phosphorus.
\end{abstract}

Keywords: DFT, bio-batteries, sodium ion batteries

Received: January-27-2021, Accepted: February-19-2021, https://doi.org/10.14447/jnmes.v24i1.a01

\section{ABBREVIATIONS}

$E_{a d} \quad$ Adsorption energy without sodium

$E_{t o t} \quad$ Total energy of the adsorbed configuration

$E_{\text {Pyrazine }}$ The energy of pyrazine in the same cell

$E_{n a} \quad$ Energy of standalone sodium ion

$n_{n a} \quad$ Number of sodium ions

$E_{\text {Номо }}$ Energy of the highest occupied molecular orbital

$E_{L U M O} \quad$ Energy of the lowest unoccupied molecular orbital

$E_{H L G}$ Energy difference between the HOMO and LUMO

orbital

$E_{a d s}$ The adsorption energy of the configuration with sodium ion

$E_{\text {def }} \quad$ Deformation energy

$E_{\text {rig }} \quad$ Rigid energy

\section{INTRODUCTION}

The fast development of electric vehicles, renewable energy for powering our industries, and various portable electronic devices provide a strong incentive to develop high energy density electric batteries. Despite their success in portable electronic devices, traditional lithium-ion batteries struggle to meet the demands of electric vehicles and large-scale energy storage due to high production costs and uneven geographical distribution [1,2]. Sodium ion batteries (SIBs) have been attracting attention as an alternative to lithium-ion batteries (LIBs) for long-term and large-scale energy storage due to their high abundance, cheap cost as compared to LIBs, safety, considerably high energy density, and lightweight [3-5]. SIBs work on the same principle that LIBs work on: the migration of sodium ions from cathode to anode during discharging and back to the cathode from anode during the charging process. However, the SIBs' practical applications are limited by their poor rate capability and low reverse capability. The large size of a $\mathrm{Na}^{+}$ion as compared to that of $\mathrm{Li}^{+}$ion leads to high volumetric change, pulverization of anode materials, and slow diffusive capability [6-8]. One of the most significant issues that are hindering the wide adoption of SIBs is the anode that is used for LIBs does not work well for SIBs, such as graphite which shows poor thermal stability, the formation of unwanted complexes such as $\mathrm{NaC}_{70}$ instead of $\mathrm{NaC}_{6}$ and formation of insufficient layer for the sodium intercalation $[9,10]$. These issues also cause problems in the formation of a stable solidelectrolyte interface (SEI); it is essential to find better electrodes to achieve satisfactory efficiency.

Many alternate anode materials on discharge are being studied for use in SIBs [11-13]. Many polymer-based biobatteries and bio-electrodes are also attracting attention due to the properties they possess, such as high energy density, easily usable at room temperature, non-polluting, bio-degradable, and safety [13-16]. Many new bio-electrodes are also being identified as a replacement for the graphite anode, which is widely used in LIBs and also in some SIBs, due to their low cost and environment-friendly nature [17-19]. However, low redox stability, very high solubility in battery electrolyte and extremely low electronic conductivity limit their wide adoption [20, 21]. Pyrazine based polymers have been known to provide high-performance capabilities of $220 \mathrm{mAhg}^{-1}$ at 50 
$\mathrm{mA} \mathrm{g}^{-1}$, which corresponds to a high energy density of $440 \mathrm{Wh}$ $\mathrm{kg}^{-1}$, and it still retains $100 \mathrm{mAh} \mathrm{g}^{-1}$ at $10 \mathrm{Ag}^{-1}$, even after 50,000 cycles [22]. Pyrazine improves the rate capability, cycling performance and has a high-temperature tolerance for LIBs; it can be easily synthesized and has a modest cost [23, 24]. The Sodium ion batteries have a similar working method like Lithium-ion batteries, consisting of two main steps: the intercalating of sodium ions at the high potential in the carbonbased anode and then inserting of sodium ion in pores at low potential $[45,46]$. The mechanism is studied for the carbonbased ring.

In this paper, a density functional theory (DFT) study of pyrazine doped with boron, fluorine, phosphorous, and sulphur is carried out to establish its suitability as a negative electrode in SIBs. Important properties, such as adsorption energies, adsorption sites, molecular orbital, the density of states, and electron density differences, are studied.

\section{COMPUTATIONAL DETAILS}

The adsorption energy, charge transfer, density of state and bond length was studied under optimized geometrical structures by density functional theory (DFT) using a DMol 3 Package in Materials Studio [27, 28]. The basis set was the generalized gradient approximation (GGA) [29] with PerdewBurke-Ernzerhof (PBE) functional to observe electronelectron exchange relation in the simulation model. The Tkatchenko and Scheffler's (TS) [30] was employed to consider the van der Waals interaction for the long-range dispersion correction of the electrons' interactions. In the sodium-carbon system, TS scheme has been proved to provide an accurate description for the diffusion barriers and adsorption energy of the compounds which are being studied [31]. Double numerical plus polarization (DNP) and DFT semi-core pseudopods were used as basis sets and to describe core electrons, respectively. The k-points which were used to configure optimization of the periodic models were $3 \times 3 \times 1$ generated by the Monkhorst-Pack scheme for the Brillouin zone sampling [32]. The same $3 \times 3 \times 1$ supercells and
Monkhorst-Pack scheme were used to study pyrazine and Xpyrazine where $\mathrm{X}=$ fluorine, sulphur, boron, or phosphorous. A dense $8 \times 8 \times 1 \mathrm{k}$-point grid was used to calculate the density of state (DOS) for the electronic relaxation, which helped in calculating the doping locations in the compounds. The converge tolerance was set as $2 \times 10^{-5} \mathrm{Ha}$, maximum force at $4 \times 10^{-3} \mathrm{Ha} \AA^{-1}$ and maximum displacement at $5 \times 10^{-3} \AA$. The periodic boundary conditions (PBC) were applied at $3.3 \AA$ in the $\mathrm{z}$-direction to avoid contact with other layers in the crystal lattice. The amount of charge transfer between sodium and host compounds was estimated using the population analysis, which was then analyzed using Mulliken charge. The charge density difference was calculated using Castep Package [33] with a plane wave energy cutoff at $517.0 \mathrm{eV}$. The adsorption energies and possible adsorption sites were calculated using the adsorption calculator tool. The Broyden-FletcherGoldfarb-Shanno (BFGS) algorithm was used to optimize the geometrical structure. The formula,

$$
\Delta \rho=\rho_{\text {tot }}-\left(\rho_{\text {pyrazine }}+\rho_{\text {sodium }}\right)
$$

was used to calculate the charge density difference where $\rho_{\text {tot }}$ $\rho_{\text {pyrazine }}$ and $\rho_{\text {sodium }}$ are the real space electronic charge of distributions of sodium adsorbed pyrazine, pyrazine, and standalone sodium, respectively.

The electrode reactions that take place are given in equations 2 and 3. P represents pyrazine, and $\mathrm{Na}$ is sodium ion, where e represents an electron. The reactions are the same for every doped pyrazine.

$$
\begin{aligned}
& \text { At positive electrode: } \mathrm{Na} \rightarrow \mathrm{Na}^{+}+\mathrm{e} \\
& \text { At negative electrode: } \mathrm{P}+\mathrm{e} \rightarrow \mathrm{P}
\end{aligned}
$$

\section{RESULTS AND DISCUSSION}

\subsection{Pyrazine doping}

(a)

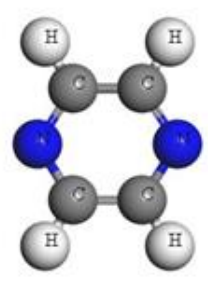

(b)

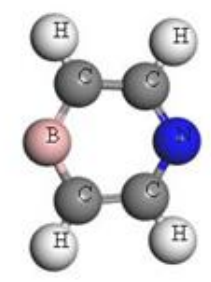

(c)

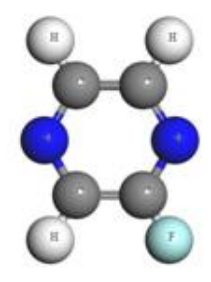

(d)

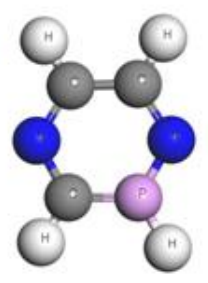

(e)

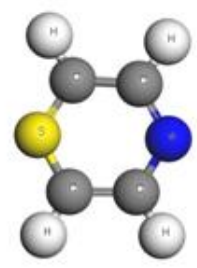

Figure 1. Optimized geometric structures of pyrazine and heteroatom doped pyrazine. (a) P optimized pyrazine. (b) PB optimized boron-doped pyrazine. (c) PF optimized fluorine-doped pyrazine. (d) PP optimized phosporous doped pyrazine. (e) PS optimized sulphur doped pyrazine 
The geometrically optimized structures of pyrazine and its various heteroatom doped configurations are shown in Figure 1. The position in the pyrazine ring and hydrogen replacement for the doping was selected through the optimization geometries and the suitable adsorption sites, which were observed during the optimized geometry and adsorption energy locator. Pyrazine, boron-doped pyrazine, fluorine doped pyrazine, phosphorous doped pyrazine and sulphur doped pyrazine are denoted as $\mathrm{P}, \mathrm{PB}, \mathrm{PF}, \mathrm{PP}$ and PS respectively. The most stable doped sites for each atom are shown in Figure 1. Boron and sulphur replaced nitrogen on the opposite sides of the ring, as shown in Figures 1(a) and (e), where as the best doping site for fluorine was replacing the hydrogen atom and phosphorus replaced the carbon atom in the ring. As the electronic configuration of boron is $2 s^{2} 2 p^{1}$, whereas the electronic configuration of a nitrogen atom in the ring is $2 \mathrm{~s}^{2} 2 \mathrm{p}^{3}$, two extra pi-bond electrons valence is emptied in the ring, which creates for a movement of the Fermi level towards the conduction band, as shown in PDOS of PB. The addition of nitrogen-based doped compounds have known to improve performance for an energy-related application, and it also introduces new functionalities when it is introduced [3436]. The functionality of our configuration has also improved the performance of the batteries due to the electronic configuration of nitrogen.

The electronic configuration for fluorine is $2 s^{2} 2 p^{5}$, which limits the hybridization of fluorine to an sp-type, so the fluorine bonds with carbon having a valence shell35. The sulphur and phosphorus go towards a pyramidal-like bonding with the neighboring carbon atoms, and they are both pulled out of the plane as shown in their crystal lattice in Figures 2 (c) and (e). The $\mathrm{P}$ is a fairly empty crystal lattice, whereas $\mathrm{PB}$ and PF have highly dense crystal lattices. While every pyrazine structure exhibits a cubic crystal lattice, the number of compounds is different due to the attraction of these specific compounds like PB and PF towards a highly dense structure. The PS has a similar structure to pyrazine, which is due to distortion in the ring of pyrazine where phosphorous is present The case is also identical for sulphur which has a highly distorted lattice, but it is still denser than PP. This distortion results in a significantly empty crystal lattice. The most stable lattices are also the ones that are most dense, as shown in Figures 2(b), (c), and (e). It can be seen from Figure 1(a) that the $\mathrm{P}$ crystal lattice is extremely stable, and different heteroatom doped affect its stability. The more stable structure also results in a more stable cyclic capacity of the sodium ion battery. (a)

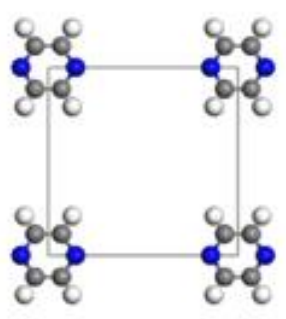

(b)

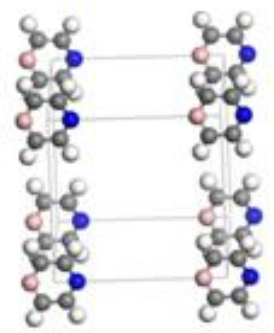

(c)

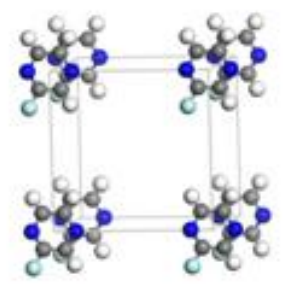

(d)

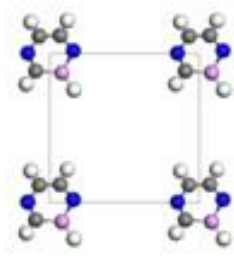

(e)

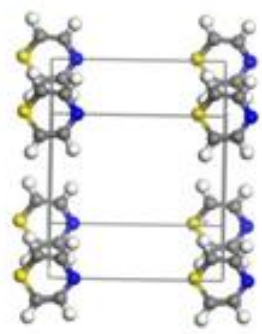

Figure 2. Crystal lattices. (a) Crystal lattice of optimized P. (b) Crystal lattice of optimized PB. (c) The crystal lattice of optimized PF. (d) The crystal lattice of optimized PP. (e) The crystal lattice of optimized PS

\subsection{Sodium ion adsorption sites and adsorption energies}

To find the most favourable adsorption sites for the sodium ion on each structure, the adsorption energy, and close contacts are shown in Table 1 and Figure 3, respectively. As seen in Figure 3, while the sodium ions interact with the pyrazine ring, the most favored adsorption sites are near the doped atoms in the ring. Sodium ions also favour the hollow sites for their position, whether it is in the rings hollow site or away from the ring. To further investigate the sodium ion adsorption on the various doped pyrazine structures, we calculate the adsorption energy as,

$$
E_{a d}=E_{\text {total }}-\left(E_{\text {pyrazine }}+n E_{n a}\right) / n_{n a}
$$

The above equation implies the more negative adsorption energy is, the more attraction is of sodium ions towards the pyrazine and its doped structures. For each structure, the simulations were carried out and the results are shown in Table 1.
Table 1. Favourable adsorption sites in the compounds

\begin{tabular}{ccc}
\hline Compound & $\begin{array}{c}\text { Favoured adsorption } \\
\text { site }\end{array}$ & $\begin{array}{c}\text { Adsorption energy } \\
(\mathbf{e V})\end{array}$ \\
\hline Pyrazine & $\mathrm{C} 2$ & -2.05 \\
PB & $\mathrm{B}$ & -2.735 \\
PF & F & -0.54 \\
PP & N1 & -0.297 \\
PS & S & -0.28 \\
\hline
\end{tabular}

The pyrazine and PB exhibit high adsorption energies of 2.05 and $-2.735 \mathrm{eV}$, respectively. From Figure 3(b), the adsorbed sodium ion remains stable in its position, which indicates that the sodium ion favours the boron adsorbed site. $\mathrm{PF}$ is in intermediate with an adsorption energy of $-0.54 \mathrm{eV}$. The least stable and also the ones with the least adsorption energy are PP and PS, which can be seen from Figures 1(d) and (e) where they show a relatively distorted structure. These observations are further reinforced from Figure 3, where we can see the adsorbed sodium ions on various compounds. PB shows a fairly stable structure, whereas PP and PS are not as stable. This also explains why in PS, the sodium ion is present in between the ring. It is due to the fact that the adsorption 
energy of a single site is not enough to attract the sodium ion towards itself. The bond lengths noted in Table 2 also help explain the low and high adsorption energies that are observed in Table 1. The longer the bond is of a configuration, the lower is the adsorption energy of that specific configuration. Furthermore, carbon provides an attractive site for the adsorption of Sodium ion [37]. The interfacial attraction, as it is the most in PB and sodium ion, also increases adsorption energy [38, 39].

The close contacts of the sodium ions are shown in Figure 3. These close contacts help us in understanding where the sodium ions are most likely to get adsorbed on the configuration. As we can see, there are multiple close contacts in Figure 3. The pink dotted line represents the close contacts. The adsorption energies and the bond lengths are shown in Table 1 and Table 2, respectively, combined with the help of close contacts, which help us in evaluating the best adsorption site for the sodium ions in the configuration. It can also see from the figure that the sodium ion prefers a hollow site, whether it is in the ring or away from the whole configuration. (a)

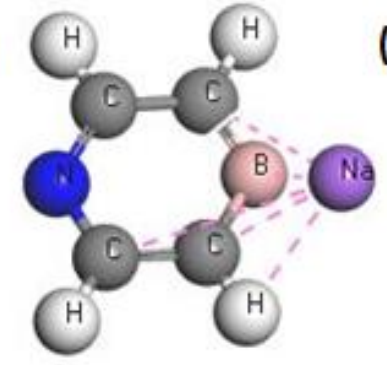

(b)

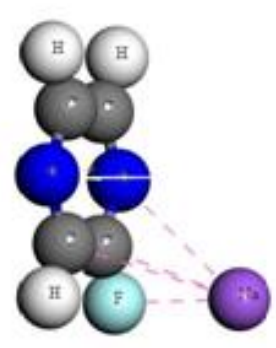

(c)

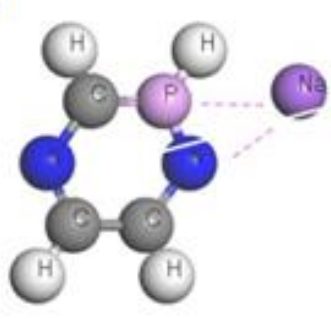

(d)

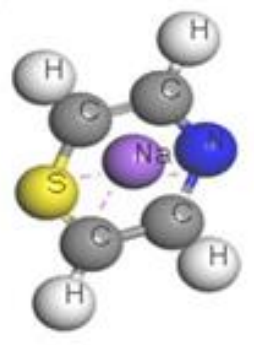

Figure 3. Close contact of sodium ion with the configured structures. (a) PB with sodium ion. (b) PF with sodium ion. (c) PP with sodium ion. (d) PS with sodium ion. The pink dotted lines represent the close contacts

Table 2. Bond lengths

\begin{tabular}{ccc}
\hline Compound & Specific bond & Bond length $(\AA)$ \\
\hline Pyrazine & N1-C1 & 1.321 \\
PB & B-Na & 1.731 \\
PF & F-Na & 2.015 \\
PP & P-Na & 1.973 \\
PS & S-Na & 2.034 \\
\hline
\end{tabular}

The symbols for the elements are used to represent the two elements that the bond is measured between. The bond lengths shown in Table 2 reaffirm our findings that the higher adsorption energy leads towards a shorter bond length [40]. The different results are obtained in the case of PP. The bond length observed is $1.973 \AA$, which is less than what is observed for PF, which has the bond length of $2.015 \AA$; Even though the adsorption energy of PF is higher than PP at -0.54 and -0.297 $\mathrm{eV}$, respectively. This can be explained by observing the close contacts, which are shown in Figure 3. There are only two close contacts observed for the PP as shown in Figure 3(c), whereas three close contacts are present in the case of PF as shown in Figure 3(b), the more repulsion that is created in PF due to the presence of fluorine, nitrogen and carbon results in a more lengthy bond between F-Na as observed in Table 2. In contrast, the presence of the two close contacts means less repulsion is present between $\mathrm{P}-\mathrm{Na}$, which shows a shorter bond length.

\subsection{Molecular orbital and energies}

Table 3 notes various energies of the heteroatom dopedpyrazine. The $E_{H L G}$ is the HOMO-LUMO gap energy in Table 3 which is calculated as:

$$
E_{H L G}=E_{H O M O}-E_{L U M O}
$$

Table 3. Energies of the heteroatom doped-pyrazine

\begin{tabular}{cccccccc}
\hline & $E_{\text {HOMO }}$ & $E_{\text {LUMO }}$ & $E_{\text {HLG }}$ & $E_{\text {tot }}$ & $E_{\text {ads }}$ & $E_{\text {def }}$ & $E_{\text {rig }}$ \\
\hline & $\mathrm{eV}$ & $\mathrm{eV}$ & $\mathrm{eV}$ & $\mathrm{kJ} / \mathrm{mol}$ & $\mathrm{kJ} / \mathrm{mol}$ & $\mathrm{kJ} / \mathrm{mol}$ & $\mathrm{kJ} / \mathrm{mol}$ \\
$\mathrm{PB}$ & -0.118 & -0.065 & 0.053 & 4.012 & -72.5 & -67.72 & -4.774 \\
$\mathrm{PF}$ & -0.094 & -0.083 & 0.011 & 6.28 & -11.46 & -7.23 & -4.23 \\
$\mathrm{PP}$ & -0.15 & -0.07998 & 0.07002 & 5.85 & -286.86 & -281.84 & -5.02 \\
$\mathrm{PS}$ & -0.107 & -0.056 & 0.051 & 6.127 & -190.70 & -185.966 & -4.73 \\
\hline
\end{tabular}


(a)

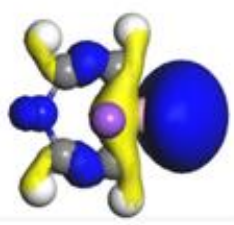

(e)

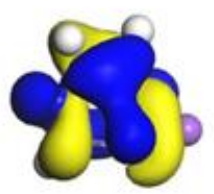

(b)

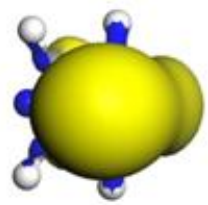

(f)

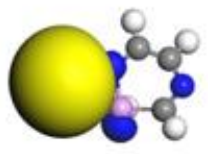

Low electron density (c)

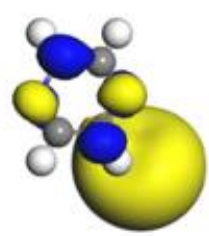

(g)

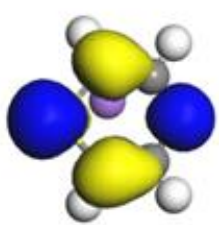

(d)

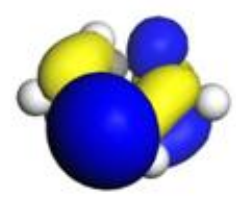

(h)

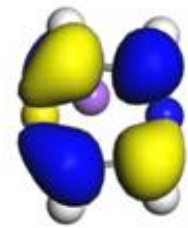

High electron density

Figure 4. Molecular orbital of heteroatom doped-pyrazine. (a) HOMO PB. (b) LUMO PB. (c) HOMO PF. (d) LUMO PF. (e) HOMO PP. (f) LUMO PP. (g) HOMO PS. (h) LUMO PS

The most $\mathrm{E}_{\mathrm{HLG}}$ is observed for $\mathrm{PF}$, which is $0.011 \mathrm{eV}$, whereas the highest $\mathrm{E}_{\mathrm{HLG}}$ is observed for PP, which is 0.07002 $\mathrm{eV}$. It means that it is easiest for PF from all the doped atoms to move electrons from the lowest orbital to the highest orbital. This also means that PF will also have the highest conduction of all the doped Pyrazine compounds [41-43]. It also means that intercalation and de-intercalation are easiest for the fluorine-doped pyrazine. The second highest $\mathrm{E}_{\mathrm{HLG}}$ is observed for $\mathrm{PB}$ at $0.053 \mathrm{eV}$, and the third one has $0.051 \mathrm{eV}$ as $\mathrm{E}_{\mathrm{HLG}}$. There is not a huge gap between the PB and PS as there is a gap between PF and PP. The opposite trend is observed for the total energy noted in Table 3 . The PF has the highest total energy at $6.28 \mathrm{eV}$. This effect is not predominant in all the heteroatom doped configurations, as can be seen in Table 3. There are wild fluctuations in $E_{a d s}$ and $E_{d e f}$ of PF as compared to the other configurations. This means that the intercalation and de-intercalation of PF are also easy as compared to the others in Table 3. This is due to a huge gap between the deformation energy of $\mathrm{PF}$, which is only $7.23 \mathrm{~kJ} / \mathrm{mol}$, compared to $\mathrm{PP}$, which has the highest deformation energy, $281.84 \mathrm{~kJ} / \mathrm{mol}$ in Table 3 [44].

In all the HOMO and LUMO of the heteroatom dopedpyrazine, the low electron density is where the sodium ion is present. As pyrazine is an N-type compound, it means it accepts electrons from the lithium ions. When lithium is reduced by pyrazine, it accepts electrons into its LUMO. This negative charge is stored in the backbone of the organic part of the complex, and it is balanced by the $\mathrm{Na}^{+}$present in the structure [45].

The low electron density places are represented by sodium ions in both HOMO and LUMO. This is due to the electron deficiency that is present in sodium ion that is present after it donates the electrons and becomes electron positive. The nature of pyrazine is that it is an electron acceptor, which creates a great and complementary combination of electron donor and electron acceptor, which contributes towards the stability of all the configurations in all heteroatom dopedpyrazine.

\subsection{Charge density difference and PDOS}

To evaluate the charge transfer between heteroatom dopedpyrazine and sodium ion, the charge density difference is shown in Figure 5. In PB, shown in Figure 5(a), the charge is accumulated on the Pyrazine ring, and a charge is depleted from the sodium ion. This is due to pyrazine, which is electrondeficient, and its ability to attract electrons towards the ring. The sodium ion donates charges to the carbon in the pyrazine ring, which results in the sodium ion being further being ionized and being depleted of its charge. When boron is introduced to the configuration, as boron has one electron less than carbon which it replaces in the configuration, the pyrazine ring becomes electron deficient. (a)

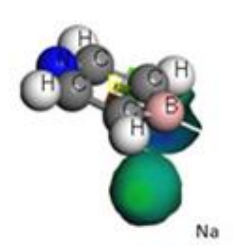

(b)

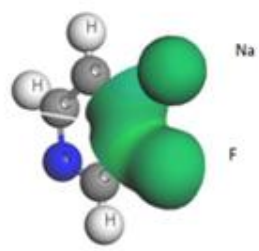

(c)

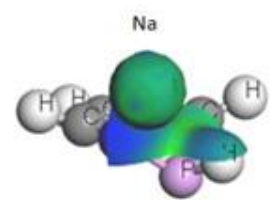

(d)

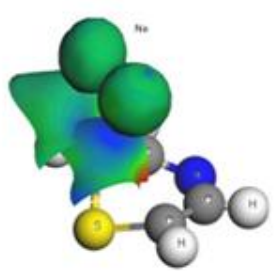

Charge depletion

Charge accumulation

Figure 5. Charge density difference plots of hetreroatom doped-pyrazine. (a) PB. (b) PF. (c) PS. (d) PP. 

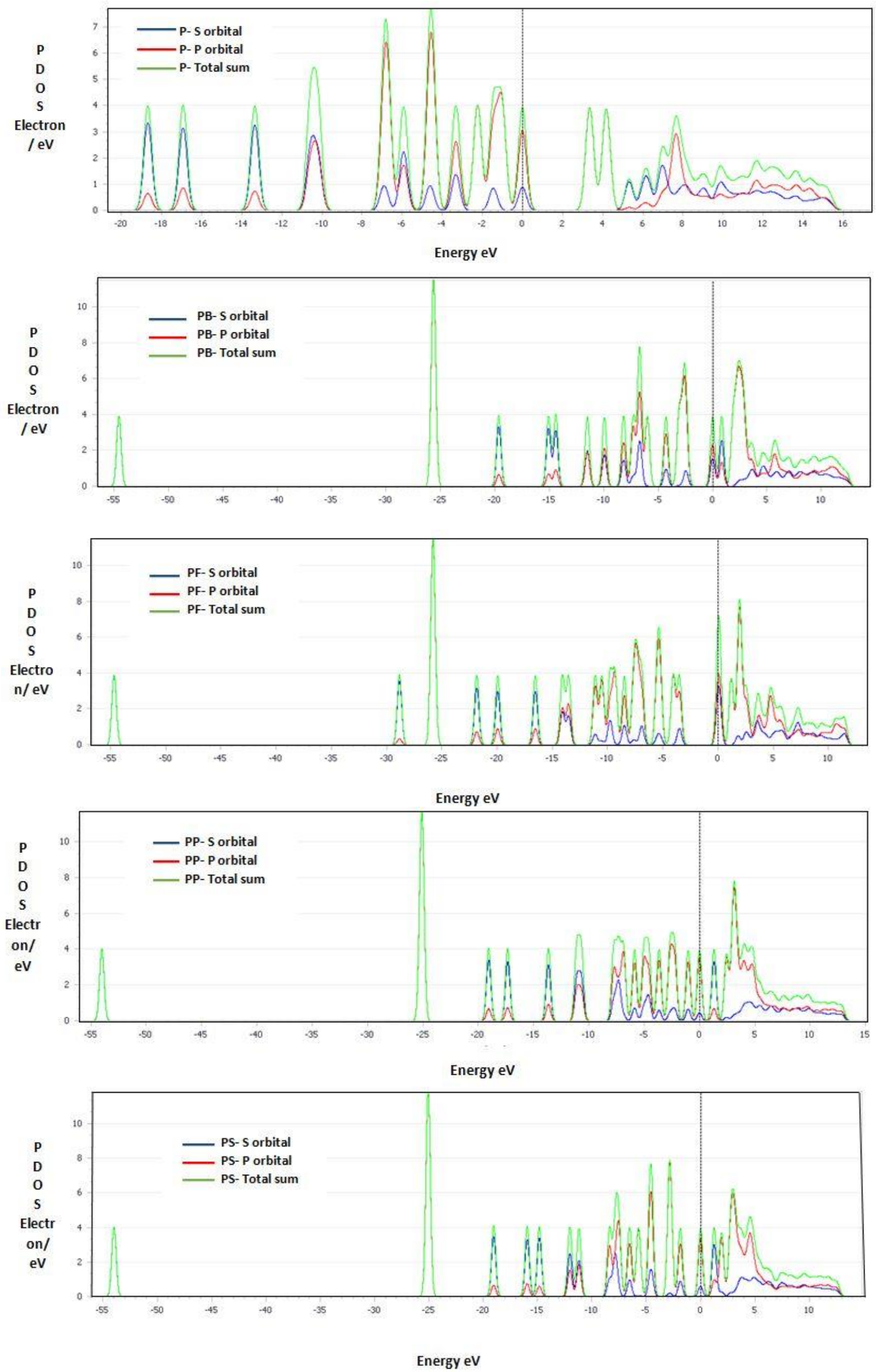

Figure 6. Partial density of states (PDOS) of heterodoped atom-pyrazine. (a) pyrazine. (b) PB. (c) PF. (d) PP. (e) PS 
In Figure 5(b), PF charge density difference plot is shown. There is a strong interaction between fluorine and sodium ion. This is due to the electronegative nature of fluorine and electron-donating nature of Sodium ion. As fluorine replaces positive hydrogen in the pyrazine ring, the fluorine also depletes the charge it has towards the highly negative carbon in the ring. $2 s^{2} 2 p^{5}$ electron configuration of fluorine leads to sp-type hybridization of fluorine to bond with the negative carbon atom in the pyrazine ring. While the charge density difference plots of $\mathrm{PB}$ and $\mathrm{PF}$ show that the sodium ion is highly depleted, in the case of PS and PP, the depletion is not as acute. In PP, this is due to replacement of the nitrogen atom with phosphorous which is not as much electronegative as fluorine. The charge accumulation is hinted towards the carbon atoms in both PP - Figure 5c) and PS - figure 5d) configurations.

To understand the adsorption of sodium ion on various configurations, we evaluate the PDOS of these configurations. The Fermi level is represented at 0 with a dotted line in Figure 5. The peaks of all the S-orbital are observed before the Fermi level in Figure 5. This shows that sodium ion is fully ionized as it only contributes to the conduction band. The peaks for the P-orbital, shown by the red line, before the Fermi level are observed only in pyrazine and PS as shown in Figure 5(a) and (e), respectively. The peak level in PDOS for the P-orbital after the Fermi level is observed in $\mathrm{PB}, \mathrm{PF}$, and $\mathrm{PP}$ as shown in Figure 5(b), (c), and (d), respectively. The peak for the Porbital observed before shows poor adsorption of the sodium ions, while the peak after the Fermi level shows good adsorption of the sodium ion.

The S-orbital peak observed for pyrazine does not match the peaks of the P-orbital peak of PB, PF, and PP as can be seen from Figures 5(b), (c), (d), and (e), respectively. This means that the strong adsorption interaction of sodium ion is caused by the electrostatic interactions. The overlapping of the $\mathrm{S}$ and P-orbital in Figure 5(e) shows the effect of localized structure effect of sulphur in the pyrazine ring, which results in a $\mathrm{sp}^{3}$ orbital overlapping with the electronegative carbon in the ring, forming a covalent bond $[29,44]$.

\section{CONCLUSION}

Pyrazine doped with different atoms has been evaluated as a possible candidate for the anode in the sodium ion batteries (SIBs) using DFT computation. Graphene doped with atoms of boron, fluorine, phosphorous, and sulphur showed how such molecules interacted with the sodium ion. Fluorine and bromine doped pyrazine showed the highest adsorption energy and formed a stable configuration with the sodium ion at the anode. The presence of sulphur and phosphorous in the ring altered the structure of the ring, which leads to less charge depletion in their configuration. The highly electronegative atoms formed a more stable but less easily intercalated and deintercalated with sodium ion.

\section{REFERENCES}

[1] Fang, Y., Yu, X.Y., Lou, X.W., Advanced Materials, 30 (21) 1706668 (2018). https://doi.org/10.1002/adma.201706668

[2] Bi, L., Li, X., Liu, X., Zheng, Q., Lin, D., ACS Sustainable Chem. Eng., 7, $7693 \quad$ (2019). https://doi.org/10.1021/acssuschemeng.8b06385
[3] Kundu, D., Talaie, E., Duffort V., and Nazar, L.F., Angew. Chem. Int. Ed., 54 (11) (2015). https://doi.org/10.1002/anie.201410376

[4] Palomares, V., Serras, P., Villaluenga, I., Huesco, K.B., Carretero-Gonzalez, J., Rojo, T., Energy Environ Sci., 5 (2012). https://doi.org/10.1039/C2EE02781J

[5] Chen, S., Feng, F., Ma, Z.F., Compos, Z.F., Commun., 22, 100447 (2020).

[6] Slater, M.D., Kim, D., Lee, E., Johnson, C.S., Adv. Funct. Mater., 23, 1200691 (2013).

https://doi.org/10.1002/adfm.201301540

[7] Ding, X., Liu, Y., Progress in Natural Science: Materials International, $\quad 30 \quad$ (2), $153 \quad$ (2020). https://doi.org/10.1016/j.pnsc.2020.01.016

[8] Zheng, X.Y., Bommier, C., Luo, W., Jiang, L., Hao, Y., Huang, Y., Energy Storage Mater., 16, 16 (2019). https://doi.org/10.1016/j.ensm.2018.04.014

[9] Asher, R.C., Wilson, S.A. (1958). Nature, 181(4606): 409-410. https://doi.org/10.1038/181409a0

[10] Nayak, P.K., Yang, L., Brehm, W., Adelhelm, P., Batteries \& Supercaps 2(1) (2018). https://doi.org/10.1002/batt.201800034

[11] Walter, M., Kovalenko, M.V., Kravchyk, K.V., New Journal of Chemistry., 44: 1677 (2020). https://doi.org/10.1039/C9NJ05682C

[12] Liu, J., Xu, Y.G., Kong, L.B., Solid State Ionics, 355, 115416 (2020). https://doi.org/10.1016/j.ssi.2020.115416

[13] Xiao, B., Rojo, T., Li, X., Chem-Sus-Chem., 12, 1801879 (2018). https://doi.org/10.1002/cssc.201801879

[14] Alday, P.P., Barros, S.C., Alves, R., Esperança, J. M.S.S. Navarro-Segarra, M., Sabaté, N., Silva, M.M. and Esquivel, J.P., Advanced Sustainable Systems, 4 (2), 2070003 (2020). https://doi.org/10.1002/adsu.202070003

[15] Suarez, A.M.N., Gonzales, J.Z., Casado, N., Mecerreyes, D., Rojo, T., Martinez, E.C., Sustainable Energy Fuels, 2, 836 (2018).

[16] Kannan, A.M., Renugopalakrishnan, V., Filipek, S., Li, P., Audette, G.F., Munukutla, L., Nanosci, J. Nanotechnol., 8, 19435024 (2009).

[17] I. Arockia Mary, S. Selvanayagam, S. Selvasekarapandian, S. R. Srikumar, T. Ponraj and V. Moniha, Ionics, 25, 5839 (2019).

[18] Rajagopalan, Y. Tang, C.Jia, X.Jia and H. Wang, H, Energy Environ. Sci.., 13, 1568 (2020).

[19] Zhao, Q., Whittaker, A.K., Zhao, X.S., Materials, 11 (12), 2567 (2018). https://doi.org/10.3390/ma11122567

[20] Nokami, T., Matsuo, Y., Inatomi, N., Hojo, T., Tsukagoshi, Yoshizawa, H., Shimizu, A., Kuramoto, H., Komae, K., Tsuyama, H., Yoshida, J.I., J. Am. Chem. Soc., 134 (48), 19694 (2012) https://doi.org/10.1021/ja306663g

[21] Chen, D., Avestro, A.J., Chen, Z., Sun, J., Wang, S., Xiao, M., Erno, Z., Algaradah, M.M., Nassar, M.S., Amine, K., Meng, Y., Stoddart, J.F., Advanced Materials, 27 (18), 2907 (2015). https://doi.org/10.1002/adma.201405416

[22] Mao, M., Luo, C., Pollard, T.P., Hou, S., Gao, T., Fan, X., Cui, C., Yue, J., Tong, Y., Yang, G., Deng, T., Zhang, M., Ma, J., Suo, L., Borodin, O., Wang, C., Angew. Chem. Int. Ed., 58, $1910916 \quad$ (2019). https://doi.org/10.1002/anie.201910916 
[23] S.E. Jerng, B. Chang, H. Shin, H. Kim, T. Lee, K. Char and J.W. Choi, ACS applied materials and interfaces., 12, 32031365 (2020).

[24] Kwon, G., Lee, S., Hwang, J., Shim, H.S., Lee, B., Lee, M.H., Ko, Y., Jung, S.K., Ku, K., Hong, J., Kang, K., Joule., 2 (9), 1771 (2018). https://doi.org/10.1016/j.joule.2018.05.014

[25] B. Delley, the journal of chemical physics., 92, 1458452 (2019).

[26] Delley, B., The Journal of chemical physics, 113 (18), 7756 (2000). https://doi.org/10.1063/1.1316015

[27] Perdew, J.P., Burke, K., Ernzerhof, M., Phys. Rev. Lett., 77, 3865 (1996).

[28] Tkatchenko, A., Scheffler, M., Phys. Rev. Lett., 102, 073005 (2020). https://doi.org/10.1063/1.3213194

[29] Qiao, Y., Ma, M., Liu, Y., Li, S., Lu, Z., Yue, H., Dong, H., Cao, Z., Yin Y. and Yang, S., Journal of Materials Chemistry A., 4, 15565 (2016).

[30] Monkhorst, H.J., Pack, J.D., Physical review B, 13 (12), 5188 (1976). https://doi.org/10.1103/PhysRevB.13.5188

[31] Clark, S.J., Segall, M.D., Pickard, C.J., Hasnip, P.J., Probert, M.J., Refson, K., Payne, M.C., Zeitschrift für kristallographie, 220, 567 (2005). https://doi.org/10.1524/zkri.220.5.567.65075

[32] B.C.B, Symons, D.J. Williamson, C.M. Brooks, A.L. Wilson and P.L.A. Popelier, chemistryopen., 8, 1(2019).

[33] L. M. Zhu, A. W. Lei , Y. L. Cao , X. P. Ai and H. X. Yang., Chem. Commun., 49, 567 (2013).

[34] M. Kaur, M. Kaur and V.K. Sharma, Adv. Colloid Interface Sci., 259, 44 (2018).

[35] S.K. Singh, K. Takeyasu and J. Nakamura, Adv. Mater., 31, 1804297 (2018).
[36] P. Bhauriyal, A. Mahata and B. Phatak, J. Phys. Chem. C., 122, 20312 (2018).

[37] Yu, T., Zhao, Z., Liu, L., Zhang, S., Xu, H., Yang, G., J. Am. Chem. Soc., $140 \quad$ (18) $5962 \quad$ (2018). https://doi.org/10.1021/jacs.8b02016

[38] Xie, X., Ao, Z., Su, D., Zhang, J., Wang, G., Adv. Funct. Mater., 25 (9), 1393 (2015). https://doi.org/10.1002/adfm.201404078

[39] Wang, W., Sun, Z., Zhang, W., Fan, Q., Sun, Q., Cui, X., Xiang, B., R. Soc. Chem., 60, 54874 (2016).

[40] Q. Sun, Y. Dai, Y. Ma, T. Jing, W. Wei and B. Huang, J. Phys. Chem. Lett., 7, 937 (2016). 26905961. https://doi.org/ 10.1021/acs.jpclett.6b00171

[41] You, Y., Manthiram, A., Adv. Energy Mater., 8 (1), 1701785 https://doi.org/10.1002/aenm.201701785

[42] Xiang, X., Zhang, K., J. Chen, Adv. Mater., 27, 1501527 (2015).

[43] Bhuvansewari, R., Nagarajan, V., Chandiramouli, R., Materials Research Express., 6 (3), 35504 (2018).

[44] T. Song, W. Yao, P. Kiadkhunthod, Y. Zheng, N. Wu, X. Zhou, S. Tunmee, S. Sattayaporn and Y. Tang, Angew. Chem. Int. Ed., 59, 1912272 (2019). https://doi.org/10.1002/anie.201912272

[45] D.A. Stevens and J.R. Dahn, J. Electrochem. Soc., 148, A803 (2001).

[46] W.K. Pang, S. Kalluri, V.K. Peterson, N. Sharma, J. Kimpton, B. Johannessen, H.K. Liu, S.X. Dou and Z. Guo., Chem. Mater., 27, 3150 (2015). 